\title{
Absolute Proper Motions Outside the Plane (APOP)
}

\author{
Zhaoxiang $\mathrm{Qi}^{1}$, Yong Yu${ }^{1}$, Richard L. Smart ${ }^{2}$, Mario G. Lattanzi ${ }^{2}$, \\ Zhenghong Tang ${ }^{1}$, Beatrice Bucciarelli ${ }^{2}$, Alberto Vecchiato ${ }^{2}$, \\ Alessandro Spagna ${ }^{2}$ and Brian J. McLean ${ }^{3}$ \\ ${ }^{1}$ Shanghai Astronomical Observatory, CAS, 80 Nandan Road, 200030 Shanghai, China \\ ${ }^{2}$ Osservatorio Astronomico di Torino, Strada Osservatorio 20, 10025 Pino Torinese, TO, Italy \\ ${ }^{3}$ Space Telescope Science Institute, 3700 San Martin Drive, Baltimore, MD 21218, USA
}

\begin{abstract}
Most of the discovered exoplanets are close to our sun. Usually their host star is with large proper motions, which is an important parameter for exoplanet searching. The first version of absolute proper motions catalog achieved based on Digitized Sky Survey Schmidt plate where outside the galactic plane $|b| \geqslant 27^{\circ}$ is presented, resulting in a zero point error less than $\pm 0.3 \mathrm{mas} / \mathrm{yr}$, and the overall accuracy better than $\pm 4.5 \mathrm{mas} / \mathrm{yr}$ for objects brighter than $R_{F}=18.5$, and ranging from 4.5 to 9.0 mas $/ y$ r for objects with magnitude $18.5<R_{F}<20.5$. The systematic errors of absolute proper motions related to the position, magnitude and color are practically all removed. The sky cover of this catalog is 22,525 degree $^{2}$, the mean density is 6444 objects/degree ${ }^{2}$ and the magnitude limit is around $R_{F}=20.5$.
\end{abstract}

Keywords. Astrometry, Catalogs, Absolute proper motions, Classification

\section{Plate Data}

The Schmidt plate data are from a database (Lasker et al., 2008) which is derived from the uncompressed Digitized Sky Surveys that the Space Telescope Science Institute has created from the Palomar and UK Schmidt survey plates and made them available to the community. These data include all the basic information for each plate, such as plate scale, observation time, measured coordinates, equatorial coordinates, magnitude and classification etc.

\section{Principle of Calibration}

The calibration of absolute proper motion is based on the hypothesis that objects (stars and galaxies) with close positions, magnitudes and colors have similar systematic errors; the absolute proper motions of galaxies are always zero and not dependent on their positions on plates, magnitudes or colors. Based on this assumption and the conditions of the available plate data, the basic idea of this work is that the plates with good quality will be chosen as reference plates, and the stellar objects with good image quality on the reference plates will be used to unify the program plates to reference plates; a new kind of moving mean method is adopted to remove the Position dependent systematic Errors (hereafter P.d.E.) via stellar objects; galaxies will be chosen from the extended objects by utilizing the motion characteristics of galaxies, and then the Magnitude and Color dependent systematic Errors (hereafter M.d.E. and C.d.E.) of all objects are removed by referring to those galaxies; finally, the absolute proper motions of stellar objects are calibrated by combing all of the plate data in different epochs. 

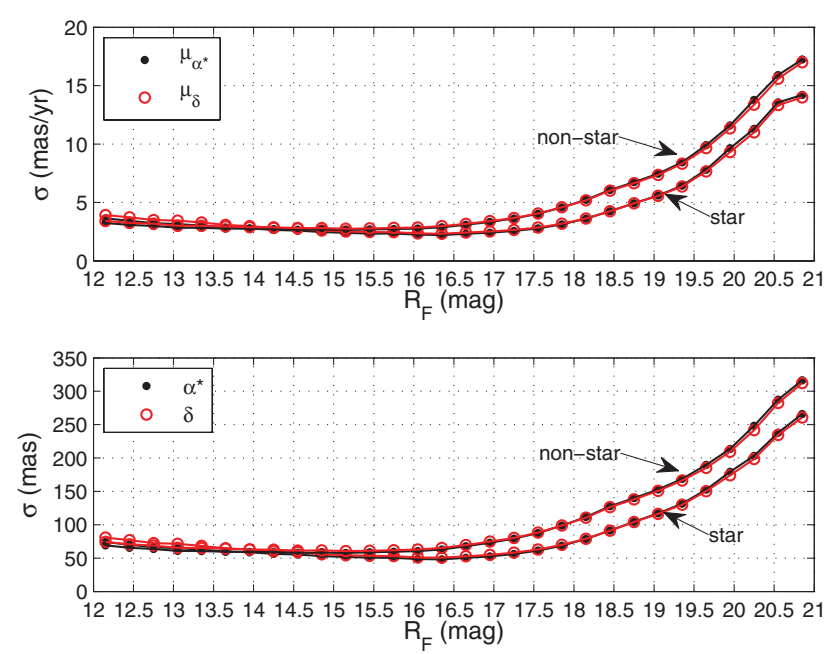

Figure 1. Shows the mean formal errors of the absolute proper motions $\left(\mu_{\alpha *}, \mu_{\delta}\right)$ and positions $(\alpha, \delta)$ of stars and non-stars as a function of $R_{F}$ magnitude in catalogue APOP. The magnitude bin for this statistics is $0.3 \mathrm{mag}$, which make sure there are at lest 100,000 objects in each bin. The marker $*$ followed the $\mu_{\alpha}$ and $\alpha$ means projecting them onto the the great circle direction (i.e times the $\cos \delta$ ). Top panel: the formal errors of absolute proper motions, the unit is milli-arcsec per year; bottom panel: the formal errors of positions, the unit is milli-arcsec.

\section{Internal Accuracy}

For each object, we could obtain the calibrated absolute proper motions and positions by fitting an equation with all its measures in different epochs. Meanwhile, we could also get the formal errors (i.e. the median standard errors) of the calibrated parameters $\left(\mu_{\alpha *}\right.$, $\left.\mu_{\delta}, \alpha, \delta\right)$, which could be as an internal check of the qualities of the catalogue APOP. The figure 1 displays the statistics.

\section{External Accuracy}

Quasi-stellar objects (QSOs) have stellar-like images and since they are extragalactic, their proper motions could be considered as zero. Thus the dispersions of their measured proper motion will be a very good measure of the zero point and overall accuracy of absolute proper motions of the stellar objects. Here we use them as an independent and direct determination of the quality of this APOP catalogue. The Large Quasar Reference Frame (LQRF) (Andrei et al., 2009) is chosen as the source list for known QSOs. The figure 2 and 3 display the statistics results.

\section{Conclusions and Future Work}

The catalogue APOP is achieved based on the Digitized Sky Survey Schmidt plate data outside of the galactic plane $|b| \geqslant 27^{\circ}$. The internal and external accuracies of this new catalogue appear to be consistent as expected. Other work (Lasker et al., 2008) has shown that there is an offset in magnitude system between stars and galaxies. To some extent, this will reduce the effect of removing the M.d.E. and C.d.E. by using galaxies. We are doing some exploratory investigation to remove these bad effects. Fortunately, the team of Guide Star Catalog II project also plans to update the photometry data. 

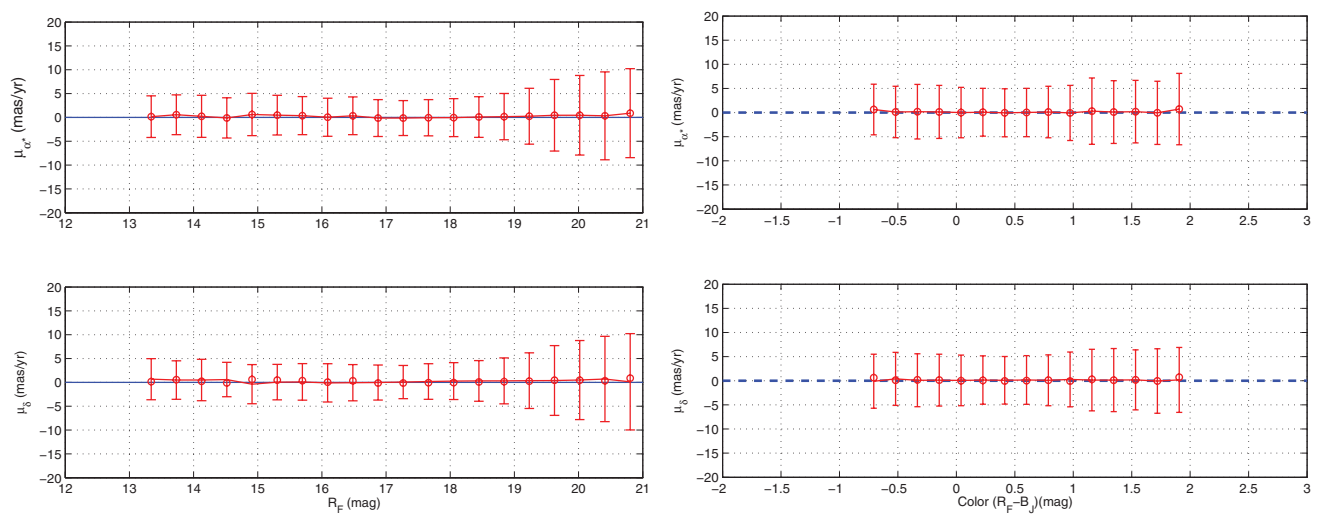

Figure 2. The left two panels show the systemic and random values of absolute proper motions $\left(\mu_{\alpha *}, \mu_{\delta}\right)$ of QSOs as a function of their magnitude. The right two panels show the absolute proper motions of QSOs as a function of their color.
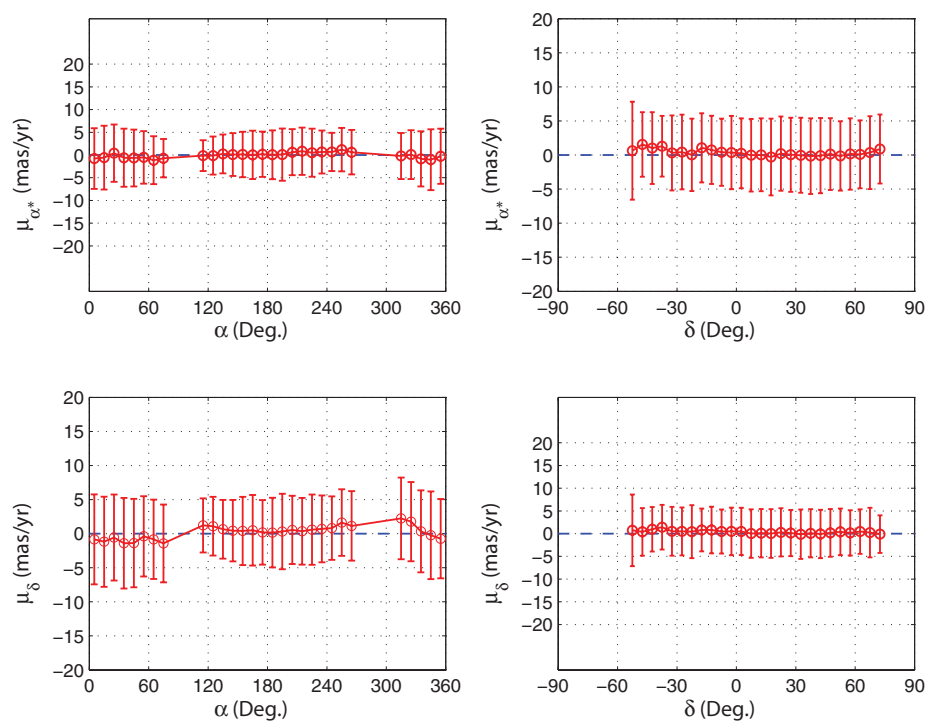

Figure 3. These four panels shows the systemic and random values of absolute proper motions of QSOs as a function of $\alpha$ and $\delta$.

\section{Acknowledgments}

This work is a joint study of the Shanghai Astronomical Observatory, the Osservatorio Astronomico di Torino and the Space Telescope Science Institute. This work is supported by grants from the National Science Foundation of China (No. 10903022) and the FP7 International Research Staff Exchange Scheme (No. 247593).

\section{References}

Lasker, et al., 2008, AJ, 136, 735-766

Andrei, et al., 2009, A\&GA, 505, 385-404 